\title{
Weighted Measurement Fusion White Noise Deconvolution Filter with Correlated Noise for Multisensor Stochastic Systems
}

\author{
Xin Wang, Shu-Li Sun, Kai-Hui Ding, and Jing-Yan Xue \\ Department of Automation, Heilongjiang University, Harbin 150080, China \\ Correspondence should be addressed to Xin Wang, wangxin@hlju.edu.cn
}

Received 27 November 2011; Revised 4 April 2012; Accepted 9 April 2012

Academic Editor: Weihai Zhang

Copyright (C) 2012 Xin Wang et al. This is an open access article distributed under the Creative Commons Attribution License, which permits unrestricted use, distribution, and reproduction in any medium, provided the original work is properly cited.

For the multisensor linear discrete time-invariant stochastic control systems with different measurement matrices and correlated noises, the centralized measurement fusion white noise estimators are presented by the linear minimum variance criterion under the condition that noise input matrix is full column rank. They have the expensive computing burden due to the high-dimension extended measurement matrix. To reduce the computing burden, the weighted measurement fusion white noise estimators are presented. It is proved that weighted measurement fusion white noise estimators have the same accuracy as the centralized measurement fusion white noise estimators, so it has global optimality. It can be applied to signal processing in oil seismic exploration. A simulation example for Bernoulli-Gaussian white noise deconvolution filter verifies the effectiveness.

\section{Introduction}

An important application background of white noise estimation is signal processing in oil seismic exploration. After the explosives buried underneath earth surface are exploded, analyzing the reflection coefficient formed by the reflections of every oil layer can be used to determine whether there is oil underground and the geometry shape of the oil field. The reflection coefficient can be described by Bernoulli-Gaussian white noise. So the white noise estimation problem can be used in the oil seismic exploration. This problem has been deeply researched by Mendel [1-3], but Mendel has not solved the system's measurement white noise estimation problem and also has not referred the problem of multisensor information fusion white noise deconvolution estimation.

In order to improve the estimation accuracy of single sensor to the white noise, in [4,5], when input noise and measurement noise are not correlated, the multisensor information 
fusion white noise deconvolution filters are put forward, respectively, by Kalman filtering method and modern time series analysis method. In [6-8], multisensor information fusion white noise optimal filter is presented for the systems with correlated noise by Kalman filtering method. In [9], modern time series analysis method and Gevers-Wouters algorithm are used to present the information fusion white noise deconvolution filter for multisensor systems with correlated noises. Thus, the solution of Riccati equation is avoided and the selftuning filter with unknown model parameters can be designed. The shortcomings of methods presented in [3-9] are to require computing the high-dimension cross-covariance matrix and the fusion accuracy is global suboptimal.

Recently, the weighted measurement fusion (WMF) method has gained great attention. Its basic principle is to weigh local sensor measurements to obtain a lowdimensional measurement equation according to some fusion criterion and then use a single Kalman filter to obtain the final fused state estimation. It can, not only reduce the computing burden greatly, but also give a global optimal estimation. This can be explained as the accuracy of WMF and the centralized measurement fusion (CMF) filter [10] is the same. Therefore, it is globally optimal. Gan and Chris [10] put forward the WMF algorithm with the assumption that all the sensors have the same measurement matrix and the measurement noises of each sensor are uncorrelated. Using the Lagrange multiplier method, the WMF algorithm is presented when the measurement noises of each sensor are correlated [11]. Ran et al. $[12,13]$ put forward the WMF algorithm when the extended measurement matrix of all the sensors has full column rank or the measurement matrices of all the sensors have the maximal right factor. Self-tuning WMF Kalman filtering algorithm is presented in the work of Gao et al. [14] and Ran and Deng [15]. In [16, 17], full-rank decomposition and weighted least square theory is used under correlated noises and different sensor measurement matrices; the WMF algorithm is presented.

However, using WMF method to solve the white noise estimation value problem of multisensor system with correlated noise and with different measurement matrices in each sensor is always a difficult issue to be solved, since the present white noise estimation theory is not suitable for WMF method. In this paper, we use the WMF algorithm to solve the white noise fusion estimation problem of multisensor systems with correlated noises and different measurement matrices. Firstly, under the assumption that the noise input matrix is of full column-rank, we present the CMF white noise estimators by the extended measurements of all sensors. They have a large computing burden due to the high-dimension measurement matrix. Further, the WMF white noise deconvolution estimators are presented to reduce the computing burden, which have the global optimality.

The paper is structured as follows. The CMF white noise deconvolution estimators are presented in Section 2. The multisensor WMF white noise deconvolution estimators are designed in Section 3. A simulation example follows in Section 4. Some conclusions are given in the end.

\section{Multisensor CMF White Noise Deconvolution Filter}

Consider the discrete time-invariant linear stochastic control systems with $L$ sensors

$$
\begin{gathered}
x(t+1)=\Phi x(t)+B u(t)+\Gamma w(t), \\
y_{i}(t)=H_{i} x(t)+v_{i}(t), \quad i=1, \ldots, L,
\end{gathered}
$$


where $x(t) \in R^{n}$ is the state, $y_{i}(t) \in R^{m_{i}}, i=1, \ldots, L$ are the measurements, $u(t) \in R^{p}$ is the known control input, $\Phi, B$, and $\Gamma$ are constant matrices with compatible dimensions. $H_{i} \in R^{m_{i} \times n}$ is the measurement matrix of the sensor $i . w(t)$ and $v_{i}(t)$ are correlated white noises with zero means, and

$$
E\left\{\left[\begin{array}{c}
w(t) \\
v_{i}(t)
\end{array}\right]\left[w^{T}(k) v_{j}^{T}(k)\right]\right\}=\left[\begin{array}{cc}
Q_{w} & S_{j} \\
S_{i}^{T} & R_{i j}
\end{array}\right] \delta_{t k}
$$

where the symbol $E$ denotes the expectation, $\delta_{t t}=1, \delta_{t k}=0(t \neq k), R_{i i}=R_{i}$, the superscript $T$ denotes the transpose. Combining $L$ measurement equations of (2.2) yields the centralized measurement equation:

$$
y^{(I)}(t)=H^{(I)}(t) x(t)+v^{(I)}(t)
$$

where

$$
y^{(I)}(t)=\left[y_{1}^{T}(t), \ldots, y_{L}^{T}(t)\right]^{T}, \quad H^{(I)}=\left[H_{1}^{T}, \ldots, H_{L}^{T}\right]^{T}, \quad v^{(I)}(t)=\left[v_{1}^{T}(t), \ldots, v_{L}^{T}(t)\right]^{T}
$$

The fusion measurement white noise $v^{(I)}(t)$ has variance matrix $R^{(I)}=\left(R_{i j}\right)_{L L}$. The correlated function of $w(t)$ and $v^{(I)}(t)$ is $S=\left[S_{1}, \ldots, S_{L}\right]$.

To convert the systems (2.1) and (2.4) into the uncorrelated system, (2.1) is equivalent to

$$
x(t+1)=\Phi x(t)+B u(t)+\Gamma w(t)+J\left[y^{(I)}(t)-H^{(I)} x(t)-v^{(I)}(t)\right]
$$

where $J$ is a pending matrix. (2.6) can be converted into

$$
x(t+1)=\bar{\Phi} x(t)+\bar{u}(t)+\bar{w}(t),
$$

where $\bar{\Phi}=\Phi-J H^{(I)}, \bar{u}(t)=B u(t)+J y^{(I)}(t), \bar{w}(t)=\Gamma w(t)-J v^{(I)}(t) . J y^{(I)}(t)$ as output feedback becomes a part of the control item. Then primary system formulae (2.1) and (2.4) are equivalent to the system formed by formulae (2.4) and (2.7). To make $E\left[\bar{w}(t) v^{(I) \mathrm{T}}(t)\right]=0$, introduce $J=\Gamma S R^{(I)-1}$ which ensures that $\bar{w}(t)$ and $v^{(I)}(t)$ are not correlated. Then variance matrix of $\bar{w}(t)$ is yielded as $\bar{Q}_{w}=\Gamma\left(Q_{w}-S R^{(I)} S^{T}\right) \Gamma^{T}$. 
Lemma 2.1 (see $[18,19]$ ). Multisensor systems (2.1) and (2.4) with correlated noise have CMF global optimal input white noise deconvolution estimators $\widehat{w}(t \mid t+N)$ and the error variance matrices $P_{w}(t \mid t+N)$ as

$$
\begin{gathered}
\widehat{w}(t \mid t+N)=0, \quad(N<0) \\
\widehat{w}(t \mid t)=S\left[H^{(I)} P(t \mid t-1) H^{(I) T}+R^{(I)}\right]^{-1} \varepsilon^{(I)}(t) \\
P_{w}(t \mid t+N)=Q_{w}, \quad(N<0) \\
P_{w}(t \mid t)=Q_{w}-S\left[H^{(I)} P(t \mid t-1)^{(I) T}+R^{(I)}\right]^{-1} S^{T} \\
\widehat{w}(t \mid t+N)=\widehat{w}(t \mid t)+\sum_{i=1}^{N} M_{w}(t \mid t+i) \varepsilon^{(I)}(t+i), \quad(N>0) \\
P_{w}(t \mid t+N)=Q_{w}-\sum_{j=1}^{N} M(t \mid t+j) Q_{\varepsilon}^{(I)}(t+j) M^{T}(t \mid t+j), \quad(N>0) \\
M_{w}(t \mid t+1)=D_{w}(t, 1) H^{(I) T}\left[H^{(I)} P^{(I)}(t+1 \mid t) H^{(I) T}+R^{(I)}\right]^{-1} \\
M_{w}(t \mid t+j)=D_{w}(t, 1)\left\{\prod_{i=1}^{j-1}\left[I_{n}-K_{f}^{(I)}(t+i) H^{(I)}\right]^{T} \bar{\Phi}^{T}\right\} H^{(I) T} \\
\times\left[H^{(I)} P^{(I)}(t+j \mid t+j-1) H^{(I) T}+R^{(I)}\right]^{-1} \\
D_{w}(t, 1)=-S K_{f}^{(I) T}(t) \bar{\Phi}^{T}+Q_{w} \Gamma^{T}-S J^{T} \\
\Psi_{p}^{(I)}(t)=\bar{\Phi}-\bar{K}_{p}^{(I)}(t) H^{(I)} \\
\bar{K}_{p}^{(I)}(t)=\bar{\Phi} K_{f}^{(I)}(t), K_{p}^{(I)}(t)=\bar{K}_{p}^{(I)}(t)+J \\
K_{f}^{(I)}(t)=P^{(I)}(t \mid t-1) H^{(I) T} Q_{\varepsilon}^{(I)-1}(t) \\
Q_{\varepsilon}^{(I)}(t)=H^{(I)} P^{(I)}(t \mid t-1) H^{(I) T}+R^{(I)} .
\end{gathered}
$$

$P^{(I)}(t+1 \mid t)$ satisfies Riccati equation:

$$
\begin{gathered}
P^{(I)}(t+1 \mid t)=\bar{\Phi}\left[P^{(I)}(t \mid t-1)-P^{(I)}(t \mid t-1) H^{(I) T}\left(H^{(I)} P^{(I)}(t \mid t-1) H^{(I) T}+R^{(I)}\right)^{-1}\right. \\
\left.\times H^{(I)} P^{(I)}(t \mid t-1)\right] \bar{\Phi}^{T}+\Gamma\left[Q_{w}-S R^{(I)-1} S^{T}\right] \Gamma^{T} \\
\widehat{x}^{(I)}(t+1 \mid t+1)=\widehat{x}^{(I)}(t+1 \mid t)+K_{f}^{(I)}(t+1) \varepsilon^{(I)}(t+1), \\
\widehat{x}^{(I)}(t+1 \mid t)=\bar{\Phi} \widehat{x}^{(I)}(t \mid t)+B u(t)+J y^{(I)}(t), \\
\varepsilon^{(I)}(t+1)=y^{(I)}(t+1)-H^{(I)} \widehat{x}^{(I)}(t+1 \mid t), \\
K_{f}^{(I)}(t+1)=P^{(I)}(t+1 \mid t) H^{(I) T}\left[H^{(I)} P^{(I)}(t+1 \mid t) H^{(I) T}+R^{(I)}\right]^{-1},
\end{gathered}
$$


Mathematical Problems in Engineering

$$
\begin{gathered}
P^{(I)}(t+1 \mid t+1)=\left[I_{n}-K^{(I)}(t+1) H^{(I)}\right] P^{(I)}(t+1 \mid t), \\
\widehat{x}^{(I)}(t \mid t+N)=\widehat{x}^{(I)}(t \mid t+N-1)+K^{(I)}(t \mid t+N) \varepsilon^{(I)}(t+N), \quad N>0, \\
P^{(I)}(t \mid t+N)=P^{(I)}(t \mid t)-\sum_{j=1}^{N} K^{(I)}(t \mid t+j) Q_{\varepsilon}^{(I)}(t+j) K^{(I) T}(t \mid t+j) .
\end{gathered}
$$

Smoothing gain has two computing methods:

$$
\begin{aligned}
& K^{(I)}(t \mid t+N)=P^{(I)}(t \mid t-1)\left\{\prod_{j=0}^{N-1} \Psi_{p}^{(I) T}(t+j)\right\} H^{(I) T} Q_{\varepsilon}^{(I)-1}(t+N), \\
& K^{(I)}(t \mid t+N)=P^{(I)}(t \mid t) \bar{\Phi}^{T}\left\{\prod_{j=1}^{N-1} \Psi_{p}^{(I) T}(t+j)\right\} H^{(I) T} Q_{\varepsilon}^{(I)-1}(t+N) .
\end{aligned}
$$

Theorem 2.2. Supposing $\Gamma$ is full column-rank matrix, for systems (2.1) and (2.4), the optimal white noise deconvolution estimator of CMF input white noise $w(t)$ is given by

$$
\begin{gathered}
\widehat{w}^{(I)}(t \mid t+N)=0, \quad(N<0), \\
\widehat{w}^{(I)}(t \mid t)=-A \widehat{x}^{(I)}(t \mid t)+C(t) .
\end{gathered}
$$

And the variance matrices of estimator errors $\widetilde{w}^{(I)}(t \mid t+N)=w(t)-\widehat{w}^{(I)}(t \mid t+N)$ are given as

$$
\begin{gathered}
P_{w}^{(I)}(t \mid t+N)=Q_{w} \quad(N<0) \\
P_{w}^{(I)}(t \mid t)=A P^{(I)}(t \mid t) A^{T}+Q \\
Q=Q_{w}-S R^{(I)-1} S^{T}
\end{gathered}
$$

defining

$$
\begin{gathered}
A=\Gamma^{+} J H^{(I)}=S R^{(I)-1} H^{(I)}, \\
C(t)=\Gamma^{+}\left(J y^{(I)}(t)\right)=S R^{(I)-1} y^{(I)}(t),
\end{gathered}
$$

where $\Gamma^{+}$is the pseudo-inverse of $\Gamma$, that is,

$$
\Gamma^{+}=\left(\Gamma^{T} \Gamma\right)^{-1} \Gamma^{T}
$$

Proof. Notice when $N<0, w(t) \perp L\left(y^{(I)}(t+N), y^{(I)}(t+N-1) \cdots\right)$, then (2.18) holds obviously. For (2.1), according to the projection theory [19], we have

$$
\Gamma \widehat{w}^{(I)}(t \mid t)=\widehat{x}^{(I)}(t+1 \mid t)-\Phi \widehat{x}^{(I)}(t \mid t)-B u(t) .
$$


From (2.11) and (2.24), we have

$$
\widehat{w}^{(I)}(t \mid t)=\Gamma^{+}\left[\bar{\Phi} \widehat{x}^{(I)}(t \mid t)+B u(t)+J y^{(I)}(t)-\Phi \widehat{x}^{(I)}(t \mid t)-B u(t)\right]
$$

which can be simplified to

$$
\widehat{w}^{(I)}(t \mid t)=\Gamma^{+}\left[-J H^{(I)} \widehat{x}^{(I)}(t \mid t)+J y^{(I)}(t)\right]
$$

Then, from the definition of (2.23), we easily obtain (2.19).

Subtracting (2.25) from (2.1) yields the error relation

$$
\Gamma \tilde{w}^{(I)}(t \mid t)=\tilde{x}^{(I)}(t+1 \mid t)-\Phi \tilde{x}^{(I)}(t \mid t) .
$$

Notice $\tilde{x}^{(I)}(t+N \mid t)=x(t)-\tilde{x}^{(I)}(t+N \mid t)$; there is a prediction error relation

$$
\tilde{x}^{(I)}(t+1 \mid t)=\bar{\Phi} \tilde{x}^{(I)}(t \mid t)+\bar{w}(t)
$$

Substituting (2.29) into (2.28) yields

$$
\Gamma \tilde{w}^{(I)}(t \mid t)=-J H^{(I)} \tilde{x}^{(I)}(t \mid t)+\bar{w}(t)
$$

And notice $\tilde{x}^{(I)}(t \mid t) \perp \bar{w}(t)$, then we have

$$
\Gamma P_{w}^{(I)}(t \mid t) \Gamma^{T}=J H^{(I)} P^{(I)}(t \mid t) H^{(I) T} J^{T}+\bar{Q}_{w}
$$

So, (2.21) holds. From (2.18), we have (2.20). The proof is completed.

Theorem 2.3. For (2.1) and (2.4), when $\Gamma$ is a full column-rank matrix, one has optimal white noise deconvolution smoothers of CMF input white noise $w(t)$ as

$$
\begin{aligned}
& \widehat{\boldsymbol{w}}^{(I)}(t \mid t+N) \\
& \quad=\widehat{w}^{(I)}(t \mid t+N-1)+E^{(I)}(t) \Psi^{(I) T}(t+N, t+1) H^{(I) T} Q_{\varepsilon}^{(I)-1}(t+N) \varepsilon^{(I)}(t+N), \quad N>0 .
\end{aligned}
$$

The error variance matrices are computed by

$$
\begin{aligned}
& P_{w}^{(I)}(t \mid t+N)=P_{w}^{(I)}(t \mid t+N-1) \\
& -E^{(I)}(t) \Psi^{(I) T}(t+N, t+1) H^{(I) T} Q_{\varepsilon}^{(I)-1}(t+N) H^{(I)} \Psi^{(I)}(t+N, t+1) E^{(I) T}(t) .
\end{aligned}
$$


Define

$$
\begin{gathered}
E^{(I)}(t)=-A P^{(I)}(t \mid t) \bar{\Phi}^{T}+Q \Gamma^{T}, \\
\Psi^{(I)}(t+N, t+N)=I_{n \prime} \\
\Psi^{(I)}(t+N, i)=\Psi_{p}^{(I)}(t+N-1) \cdots \Psi_{p}^{(I)}(i) .
\end{gathered}
$$

Proof. For (2.1), the projection theory is used, and we have

$$
\Gamma \widehat{w}^{(I)}(t \mid t+N)=\widehat{x}^{(I)}(t+1 \mid t+N)-\Phi \widehat{x}^{(I)}(t \mid t+N)-B u(t) .
$$

From (2.15), we have

$$
\begin{aligned}
\Gamma \widehat{w}^{(I)}(t \mid t+N)= & \widehat{x}^{(I)}(t+1 \mid t+N-1)+K^{(I)}(t+1 \mid t+N) \varepsilon^{(I)}(t+N) \\
& -\Phi\left[\widehat{x}^{(I)}(t \mid t+N-1)+K^{(I)}(t \mid t+N) \varepsilon^{(I)}(t+N)\right]-B u(t)
\end{aligned}
$$

From the recursive relation of (2.36), we have

$$
\widehat{w}(t \mid t+N)=\widehat{w}^{(I)}(t \mid t+N-1)+\Gamma^{+}\left[K^{(I)}(t+1 \mid t+N)-\Phi K^{(I)}(t \mid t+N)\right] \varepsilon^{(I)}(t+N)
$$

then we obtain (2.32). In fact, from (2.17), we have

$$
\begin{aligned}
\Gamma^{+}\left[K^{(I)}(t+1 \mid t+N)-\Phi K^{(I)}(t \mid t+N)\right] \\
=\Gamma^{+}\left[P^{(I)}(t+1 \mid t)\left\{\prod_{j=1}^{N-1} \Psi_{p}^{(I) T}(t+j)\right\} H^{(I)^{T}} Q_{\varepsilon}^{(I)-1}\right. \\
\left.\quad \times(t+N)-\Phi P^{(I)}(t \mid t) \bar{\Phi}^{T} \times\left\{\prod_{j=1}^{N-1} \Psi_{p}^{(I) T}(t+j)\right\} H^{(I)}{ }^{T} Q_{\varepsilon}^{(I)-1}(t+N)\right],
\end{aligned}
$$

which is simplified to

$$
\begin{aligned}
\Gamma^{+} & {\left[K^{(I)}(t+1 \mid t+N)-\Phi K^{(I)}(t \mid t+N)\right] } \\
& =\Gamma^{+}\left[P^{(I)}(t+1 \mid t)-\Phi P^{(I)}(t \mid t) \bar{\Phi}^{T}\right] \times\left\{\prod_{j=1}^{N-1} \Psi_{p}^{(I) T}(t+j)\right\} H^{(I) T} Q_{\varepsilon}^{(I)-1}(t+N) .
\end{aligned}
$$

Define $E^{(I)}(t)$ as

$$
\begin{aligned}
E^{(I)}(t) & =\Gamma^{+}\left[\bar{\Phi} P^{(I)}(t \mid t) \bar{\Phi}^{T}+\bar{Q}_{w}-\Phi P^{(I)}(t \mid t) \bar{\Phi}\right] \\
& =-\Gamma^{+} J H^{(I)} P^{(I)}(t \mid t) \bar{\Phi}^{T}+\Gamma^{+} \bar{Q}_{w} .
\end{aligned}
$$


Then we obtain (2.34). From the definition of (2.35), (2.32) is proved. Using $w(t)$ minus both sides of (2.32), and from $\widetilde{w}^{(I)}(t \mid t+N) \perp \varepsilon^{(I)}(t+N)$, (2.33) is proved. This completes the proof.

Corollary 2.4. For multisensor systems with correlated noise (2.1) and (2.4), the nonrecursive white noise smoothers are given by

$$
c \widehat{\boldsymbol{w}}^{(I)}(t \mid t+N)=\widehat{\boldsymbol{w}}^{(I)}(t \mid t)+E^{(I)}(t) \sum_{j=1}^{N} \Psi^{(I) T}(t+j, t+1) H^{(I) T} Q_{\varepsilon}^{(I)-1}(t+j) \varepsilon^{(I)}(t+j), \quad N>0 .
$$

The error variances satisfy

$$
\begin{aligned}
& P_{w}^{(I)}(t \mid t+N) \\
& \quad=P_{w}^{(I)}(t \mid t)-E^{(I)}(t)\left\{\sum_{j=1}^{N} \Psi^{(I) T}(t+j, t+1) H^{(I) T} Q_{\varepsilon}^{(I)-1}(t+j) H^{(I)} \Psi^{(I)}(t+j, t+1)\right\} E^{(I) T}(t) .
\end{aligned}
$$

Proof. (2.32) and (2.33) are iterated by $N$ times; (2.42) and (2.43) are obtained. This completes the proof.

\section{Multisensor WMF White Noise Deconvolution Estimator}

From [20], we know that any nonzero matrix $H^{(I)}$ has full-rank decomposition

$$
H^{(I)}=F H^{(I I)}
$$

where $F$ is a full column-rank matrix with the rank $r$ and $H^{(I I)}$ is a full row-rank matrix with the rank $r$, then measurement model (2.4) can be represented as

$$
y^{(I)}(t)=F H^{(I I)} x(t)+v^{(I)}(t)
$$

Given that $F$ is a full column-rank matrix, it follows that $F^{\mathrm{T}} R^{(I)} F$ is nonsingular. Then the weighted least squares (WLS) [21, 22] method is used and the Gauss-Markov estimate of $H^{(I I)} x(t)$ is yielded as

$$
\begin{gathered}
y^{(I I)}(t)=\sum_{j=1}^{L} \Omega_{i} y_{i}(t)=\left(F^{T} R^{(I)-1} F\right)^{-1} F^{T} R^{(I)-1} y^{(I)}(t), \\
{\left[\Omega_{1}, \Omega_{2}, \ldots, \Omega_{L}\right]=\left(F^{T} R^{(I)-1} F\right)^{-1} F^{T} R^{(I)-1}}
\end{gathered}
$$


then substituting (2.4) into (3.3) yields

$$
\begin{gathered}
y^{(I I)}(t)=H^{(I I)} x(t)+v^{(I I)}(t), \\
v^{(I I)}(t)=\left(F^{T} R^{(I)-1} F\right)^{-1} F^{T} R^{(I)-1} v^{(I)}(t) .
\end{gathered}
$$

The variance matrix $R^{(I I)}=E\left[v^{(I I)}(t) v^{(I I) T}(t)\right]$ of $v^{(I I)}(t)$ is given by $R^{(I I)}=\left(F^{T} R^{(I)-1} F\right)^{-1}$.

For systems (2.7) and (3.5) using standard Kalman filtering algorithm [19], we can obtain WMF Kalman estimators $\widehat{x}^{(I I)}(t \mid t)$, and its variance matrices $\mathrm{P}^{(I I)}(t \mid t)$, innovation $\varepsilon^{(\mathrm{II})}(t+j), j>0$. It is proved in [16] that the WMF Kalman filter $\widehat{x}^{(I I)}(t \mid t)$ has the global optimality; that is, it is numerically identical to the CMF Kalman filter $\hat{x}^{(I)}(t \mid t)$ if they have the same initial values.

The above WMF method can obviously reduce the computing burden since the dimension of the measurement vector for the CMF is $m \times 1, m=m_{1}+m_{2}+\cdots+m_{L}$, while that for the WMF is $r \times 1$, and $m$ is much larger than $r$ generally.

Theorem 3.1. For (2.7) and (3.5), when $\Gamma$ is a full column-rank matrix, one has WMF optimal nonrecursive smoothers of input white noise $w(t)$

$$
\begin{aligned}
& \widehat{w}^{(I I)}(t \mid t+N) \\
& =\widehat{w}^{(I I)}(t \mid t)+E^{(I I)}(t) \sum_{j=1}^{N} \Psi^{(I I) T}(t+j, t+1) H^{(I I) T} Q_{\varepsilon}^{(I I)-1}(t+j) \varepsilon^{(I I)}(t+j), \quad N>0 .
\end{aligned}
$$

The error variance matrices satisfy

$$
\begin{aligned}
P_{w}^{(I I)}(t \mid t+N)= & P_{w}^{(I I)}(t \mid t)-E^{(I I)}(t) \\
& \times\left\{\sum_{j=1}^{N} \Psi^{(I I) T}(t+j, t+1) H^{(I I) T} Q_{\varepsilon}^{(I I)-1}(t+j) H^{(I I)} \Psi^{(I I)}(t+j, t+1)\right\} \\
& \times E^{(I I) T}(t),
\end{aligned}
$$

where

$$
\begin{gathered}
E^{(I I)}(t)=-A P^{(I I)}(t \mid t) \bar{\Phi}^{T}+Q \Gamma^{T}, \\
\Psi^{(I I)}(t+N, t+N)=I_{n}, \\
\Psi^{(I I)}(t+N, i)=\Psi_{p}^{(I I)}(t+N-1) \cdots \Psi_{p}^{(I I)}(i) .
\end{gathered}
$$

If $\widehat{x}^{(I)}(0 \mid 0)=\widehat{x}^{(I I)}(0 \mid 0)$ is satisfied, then WMF is numerically equivalent to CMF, that is,

$$
\begin{array}{ll}
\widehat{w}^{(I)}(t \mid t+N)=\widehat{w}^{(I I)}(t \mid t+N) & \forall N, \forall t, \\
P_{w}^{(I)}(t \mid t+N)=P_{w}^{(I I)}(t \mid t+N) & \forall N, \forall t,
\end{array}
$$


where white noise filter is

$$
\widehat{w}^{(I I)}(t \mid t)=-A \widehat{x}^{(I I)}(t \mid t)+C(t) .
$$

Proof. From [16], when $\widehat{x}^{(I)}(0 \mid 0)=\widehat{x}^{(I I)}(0 \mid 0)$, we have

$$
\begin{aligned}
H^{(I) T} Q_{\varepsilon}^{(I)-1}(t) \varepsilon^{(I)}(t) & =H^{(I I) T} Q_{\varepsilon}^{(I I)-1}(t) \varepsilon^{(I I)}(t) \\
\Psi_{p}^{(I)}(t) & =\Psi_{p}^{(I I)}(t) \\
P^{(I)}(t \mid t) & =P^{(I I)}(t \mid t) \\
\widehat{x}^{(I)}(t \mid t) & =\widehat{x}^{(I I)}(t \mid t) .
\end{aligned}
$$

So, we have $E^{(I)}(t)=E^{(I I)}(t)$. (2.19), (2.42), and (2.43) are compared with (3.7), (3.8), and (3.12), then (3.11) are obtained.

Corollary 3.2. WMF input white noise recursive smoothers are

$$
\begin{aligned}
\widehat{\boldsymbol{w}}^{(I I)}(t \mid t+N)= & \widehat{\boldsymbol{w}}^{(I I)}(t+N-1)+E^{(I I)}(t) \Psi^{(I I) T}(t+N, t+1) H^{(I I) T} \\
& \times Q_{\varepsilon}^{(I I)-1}(t+N) \varepsilon^{(I I)}(t+N), \quad N>0 .
\end{aligned}
$$

The error variance matrices satisfy

$$
\begin{aligned}
P_{w}^{(I I)}(t \mid t+N)= & P_{w}^{(I I)}(t+N-1)-E^{(I I)}(t) \Psi^{(I I) T}(t+N, t+1) H^{(I I) T} \\
& \times Q_{\varepsilon}^{(I I)-1}(t+N) H^{(I I)} \Psi^{(I I)}(t+N, t+1) E^{(I I) T}(t),
\end{aligned}
$$

then, one has the following relation:

$$
\begin{array}{ll}
\widehat{w}^{(I)}(t \mid t+N)=\widehat{w}^{(I I)}(t \mid t+N) & \forall N, \forall t, \\
P_{w}^{(I)}(t \mid t+N)=P_{w}^{(I I)}(t \mid t+N) & \forall N, \forall t .
\end{array}
$$

Proof. From Theorem 2.3 and (3.13), we have (3.14)-(3.17). This completes the proof.

\section{Simulation Example}

Consider the multisensor discrete linear stochastic ARMA signal system

$$
\begin{gathered}
A\left(q^{-1}\right) s(t)=C\left(q^{-1}\right) w(t), \\
y_{i}(t)=H_{0 i} s(t)+\xi_{i}(t), \quad i=1,2,3, \\
A\left(q^{-1}\right)=a_{0}+a_{1} q^{-1}+a_{2} q^{-2}, \\
C\left(q^{-1}\right)=c_{0}+c_{1} q^{-1},
\end{gathered}
$$




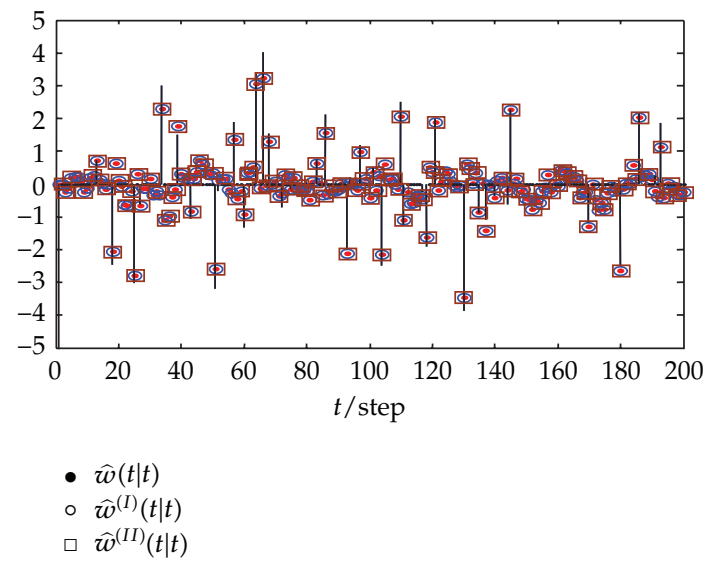

Figure 1: $w(t)$ and optimal fusion white noise filter $\widehat{w}(t \mid t), \widehat{w}^{(i)}(t \mid t), i=I, I I$.

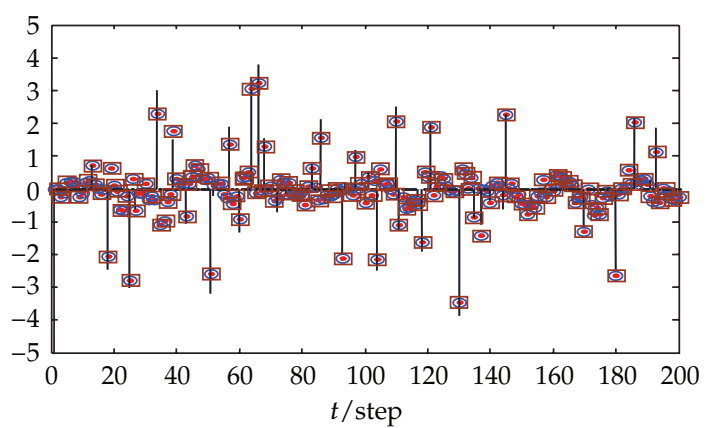

- $\widehat{w}(t \mid t+1)$

○ $\widehat{w}^{(I)}(t \mid t+1)$

$\square \widehat{w}^{(I I)}(t \mid t+1)$

Figure 2: $w(t)$ and optimal fusion white noise smoother $\widehat{w}(t \mid t+1), \widehat{w}^{(i)}(t \mid t+1), i=I, I I$.

where $s(t) \in R$ is the signal, $y_{i}(t) \in R, i=1,2,3$ are the measurement signals. $\xi_{i}(t) \in R$, $i=1,2,3$ are Gaussian white noises with zero mean and variance matrix $Q_{\xi_{i}}$. And $w(t)=$ $b(t) g(t)$, where $b(t)$ is Bernoulli white noise satisfying $b(t)=1$ if $P(b(t)=1)=\lambda$, and $b(t)=0$ if $P(b(t)=0)=1-\lambda$, where $P$ denotes probability. $b(t)$ is independent of $g(t)$, then the variance matrix of $w(t)$ is $\sigma_{w}^{2}=\lambda \sigma_{g}^{2} \cdot q^{-1}$ is the back shift operator.

Our goal is to find the optimal white noise deconvolution estimators $\widehat{w}(t \mid t+N)$, $\widehat{w}^{(I)}(t \mid t+N)$, and $\widehat{w}^{(I I)}(t \mid t+N), N=0,1,2,3$, and the corresponding error variance matrices $P_{w}(t \mid t+N), P_{w}^{(I)}(t \mid t+N)$, and $P_{w}^{(I I)}(t \mid t+N)$, to test the estimation result values of these three algorithms equal and to compare the computing burden. 


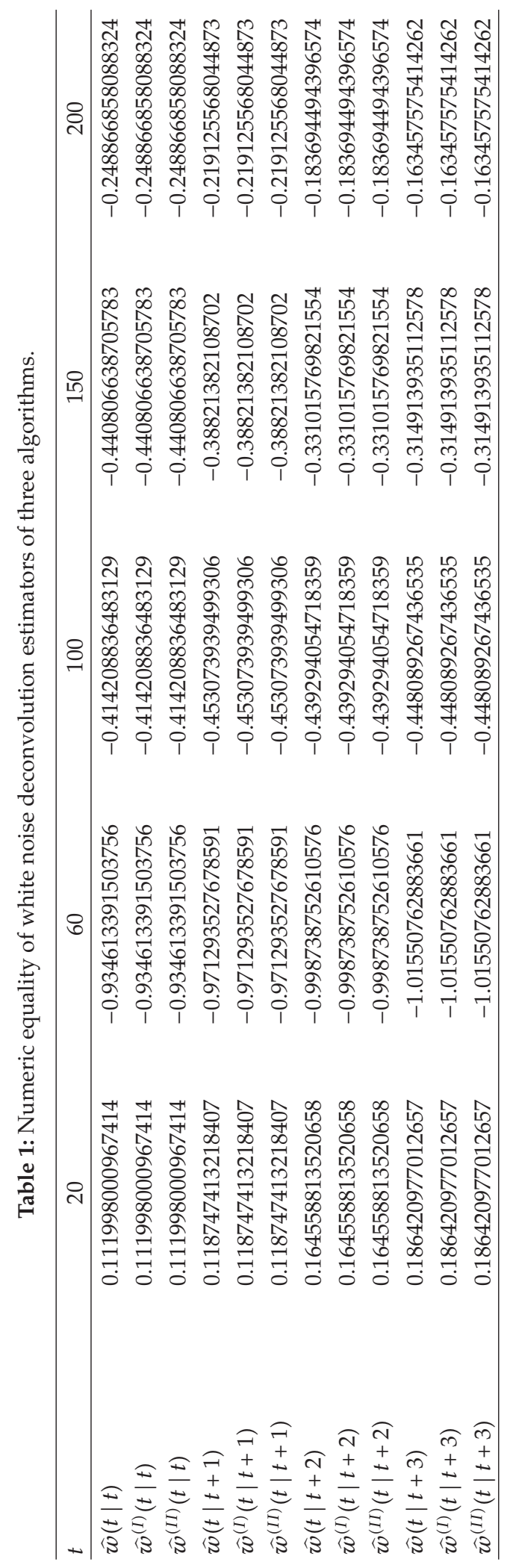




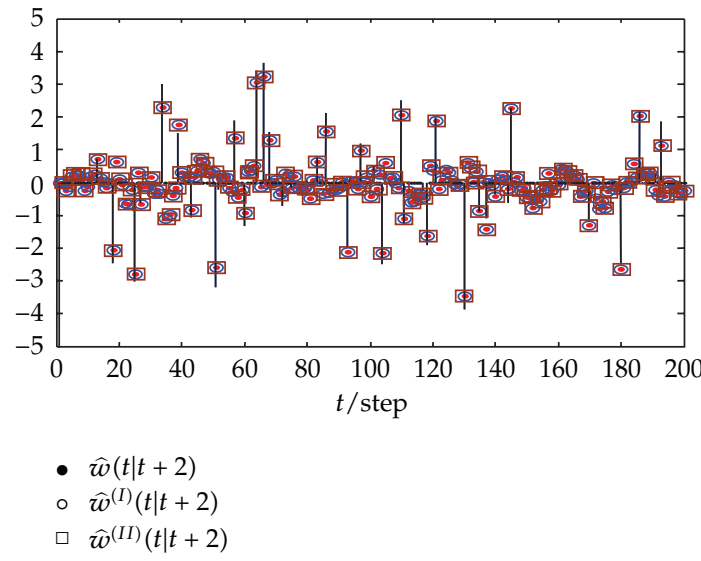

Figure 3: $w(t)$ and optimal fusion white noise smoother $\widehat{w}(t \mid t+2), \widehat{w}^{(i)}(t \mid t+2), i=I, I I$.

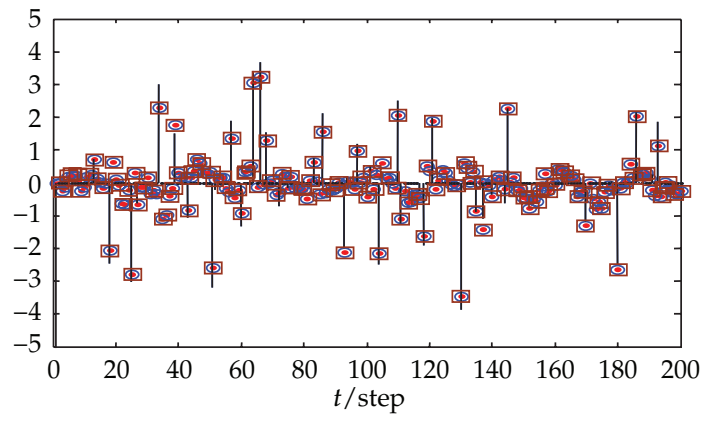

- $\widehat{w}(t \mid t+3)$

○ $\widehat{w}^{(I)}(t \mid t+3)$

$\square \widehat{w}^{(I I)}(t \mid t+3)$

Figure 4: $w(t)$ and optimal fusion white noise smoother $\widehat{w}(t \mid t+1), \widehat{w}^{(i)}(t \mid t+3), i=I, I I$.

The system (4.1) is converted to state space models

$$
\begin{gathered}
x(t+1)=\Phi x(t)+\Gamma w(t), \\
y_{i}(t)=H_{i} x(t)+v_{i}(t), \quad i=1,2,3, \\
s(t)=H x(t)+c_{0} w(t)
\end{gathered}
$$

where we define

$$
\begin{gathered}
c v_{i}(t)=H_{0 i} c_{0} w(t)+\xi_{i}(t), \\
\Phi=\left[\begin{array}{ll}
-a_{1} & 1 \\
-a_{2} & 0
\end{array}\right], \quad \Gamma=\left[\begin{array}{c}
c_{1}-a_{1} c_{0} \\
-a_{2} c_{0}
\end{array}\right], \quad H=[1,0], \quad H_{i}=H_{0 i} H .
\end{gathered}
$$


Table 2: Comparison of error variance for the local and fused estimators.

\begin{tabular}{ccccccc}
\hline$t=50$ & $P_{w}^{1}(t \mid t+N)$ & $P_{w}^{2}(t \mid t+N)$ & $P_{w}^{3}(t \mid t+N)$ & $P_{w}(t \mid t+N)$ & $P_{w}^{(I)}(t \mid t+N)$ & $\begin{array}{c}P_{w}^{(I I)}(t \mid \\
t+N)\end{array}$ \\
\hline$N=0$ & 0.28086 & 0.27750 & 0.32376 & 0.14117 & 0.14117 & 0.14117 \\
$N=1$ & 0.24502 & 0.24237 & 0.24057 & 0.13067 & 0.13067 & 0.13067 \\
$N=2$ & 0.23321 & 0.23071 & 0.21924 & 0.12607 & 0.12607 & 0.12607 \\
$N=3$ & 0.23214 & 0.22963 & 0.21913 & 0.12509 & 0.12509 & 0.12509 \\
\hline
\end{tabular}

In the simulation, we set

$$
\begin{gathered}
c a_{0}=1, \quad a_{1}=0.2, \quad a_{2}=0.3, \quad c_{0}=1, \quad c_{1}=-0.6, \quad \lambda=0.25, \quad \sigma_{g}^{2}=4, \\
c Q_{\xi_{1}}=0.1, \quad Q_{\xi_{2}}=0.2, \quad Q_{\xi_{2}}=0.4, \quad H_{01}=0.7, \quad H_{02}=1, \quad H_{03}=1.2 .
\end{gathered}
$$

So

$$
H^{(I)}=\left[\begin{array}{ccc}
0.7 & 1 & 1.2 \\
0 & 0 & 0
\end{array}\right]^{T}
$$

It is not full column rank, so Hermite standard model [20] can be used to compute the full-rank decomposition of $H^{(I)}, H^{(I)}=F H^{(I I)}$, where

$$
F=\left[\begin{array}{lll}
0.7 & 1 & 1.2
\end{array}\right]^{T}, \quad H^{(I I)}=\left[\begin{array}{ll}
1 & 0
\end{array}\right]
$$

The simulation results are presented by Figures 1, 2, 3 and 4, Tables 1 and 2. In Figures $1,2,3$ and 4 , the $y$-coordinates of endpoints on real lines represent real values $w(t), y$ coordinates of solid round points' centers represent $\widehat{w}(t \mid t+N), N=0,1,2,3, y$-coordinates of hollow round points' centers represent $\widehat{w}^{(I)}(t \mid t+N), N=0,1,2,3$. The $y$-coordinates of hollow squares' centers represent $\widehat{w}^{(I I)}(t \mid t+N), N=0,1,2,3$. It can be seen that the results are completely unanimous.

In Figure 1, it can be seen that at some specified time, the results of these three algorithms are also completely the same. Figure 2 represents the sameness of estimation error variances of the three fusion methods. The fusion accuracy is better than local estimation accuracy of every single sensor.

These results all represent that WMF is completely equivalent to CMF, and the fusion accuracy is higher than local estimation accuracy of each single sensor.

In the other aspect, it can be seen that when the filtering algorithm of CMF is used, the dimension of $y^{(I)}(t)$ is $3 \times 1$, the Kalman filtering needs to compute the inverse matrix of a $3 \times 3$ matrix at every time, while when the WMF Kalman filtering algorithm is used, the dimension of fusion measurement $y^{(I I)}(t)$ is $1 \times 1$, then the matrix inversion computation is converted into scalar division, so the WMF method can obviously reduce the computational burden. 


\section{Conclusions}

White noise deconvolution problems have great applications background in oil seismic exploration. Under the condition that the white noise input matrix is full column-rank, the centralized measurement fusion and weighted measurement fusion white noise estimators are presented based on the projection theory, respectively. Their function equivalence has been proved. Furthermore, the proposed weighted measurement fusion white noise estimators can obviously reduce the computational burden.

\section{Acknowledgments}

This work is supported by Science and Technology Research Foundation of Heilongjiang Province, Education Department under Grant 12521420.

\section{References}

[1] J. M. Mendel, "White-noise estimators for seismic data processing in oil exploration," IEEE Transactions on Automatic Control, vol. 22, no. 5, pp. 694-706, 1977.

[2] J. M. Mendel and J. Kormylo, "New fast optimal white-noise estimators for deconvolution," IEEE Trans Geosci Electron, vol. 15, no. 1, pp. 32-41, 1977.

[3] J. M. Mendel, “Minimum variance deconvolution," IEEE Transactions on Geoscience and Remote Sensing, vol. 19, no. 3, pp. 161-171, 1981.

[4] X. Wang, Y. Li, and Z. L. Deng, "Multisensor information fusion white noise deconvolution filter based on Kalman filtering method," Journal of Natural Science of Heilongjiang University, vol. 23, no. 2, pp. 227-230, 2006.

[5] Z. L. Deng, Y. Gao, and Jianwei Ma, "Two-sensor information fusion optimal white noise deconvolution Wiener filter," Science Technology and Engineering, vol. 3, no. 3, pp. 216-218, 2003.

[6] S. L. Sun, "Multi-sensor information fusion white noise filter weighted by scalars based on Kalman predictor," Automatica, vol. 40, no. 8, pp. 1447-1453, 2004.

[7] Z. L. Deng, X. Wang, and Y. Li, "Multisensor optimal information fusion white noise deconvolution filter," Acta Electronica Sinica, vol. 33, no. 5, pp. 860-863, 2005.

[8] X. Wang, Y. Li, and Z. L. Deng, "Multisensor information fusion white noise deconvolution estimator," in Proceedings of the Control and Decision, pp. 1492-1495, chn, 2006.

[9] X. J. Sun and Z. L. Deng, "Information fusion steady-state white noise deconvolution estimators with time-delayed measurements and colored measurement noises," Journal of Electronics, vol. 26, no. 2, pp. 161-167, 2009.

[10] Q. Gan and J. H. Chris, "Comparison of two measurement fusion methods for Kalman filter based multisensor data fusion," IEEE Transactions on Aerospace and Electronic Systems, vol. 37, no. 1, pp. 273$279,2001$.

[11] L. J. Ou, H. Z. Qiu, and H. Y Zhang, "Multiple corrrelated measurement fusion algorithm and its optimality," Information and Control, vol. 34, no. 6, pp. 690-695, 2005.

[12] C. J. Ran, Y. S. Hui, L. Gu, and Z. L. Deng, "Correlated measurement fusion steady-state Kalman filtering algorithms and their optimality," Acta Automatica Sinica, vol. 34, no. 3, pp. 233-239, 2008.

[13] C. J. Ran, L. Gu, and Z. L. Deng, "Correlated measurement fusion Kalman estimators and their global optimality," Control Theory and Applications, vol. 26, no. 2, pp. 174-178, 2009.

[14] Y. Gao, W. J. Jia, X. J. Sun, and Z. L. Deng, "Self-tuning multisensor weighted measurement fusion kalman filter," IEEE Transactions on Aerospace and Electronic Systems, vol. 45, no. 1, pp. 179-191, 2009.

[15] C. J. Ran and Z. L. Deng, "Self-tuning weighted measurement fusion Kalman filtering algorithm," Computational Statistics and Data Analysis, vol. 56, pp. 2112-2128, 2012.

[16] X. Wang, Q. D. Zhu, and S. L. Sun, "Weighted measurement fusion estimation algorithm with correlated noises and its global optimality," Systems Engineering and Electronics, vol. 32, no. 10, pp. 2057-2061, 2010.

[17] X. Wang, Q. D. Zhu, and S. L. Sun, "Universal weighted measurement fusion estimation algorithm and its global optimality," Computer Engineering and Applications, vol. 46, no. 24, pp. 22-25, 2010. 
[18] Z. L. Deng, Information Fusion Filtering Theory with Applications, Harbin institute of Technology Press, Harbin, China, 2007.

[19] Z. L. Deng and X. Wang, Modeling and Estimation, Science Press, Beijing, China, 2007.

[20] R. A. Horn and C. R. Johnson, Matrix Analysis, Cambrideg University Press, Cambridge, UK, 1985.

[21] T. Kailath, A. H. Sayed, and B. Hassibi, Linear Estimation, Prentice-Hall, Upper Saddle River, NJ, USA, 2000.

[22] X. Wang and S. L. Sun, "Measurement feedback self-tuning weighted measurement fusion Kalman filter for systems with correlated noises," Journal of Applied Mathematics, vol. 2012, Article ID 324296, 16 pages, 2012. 


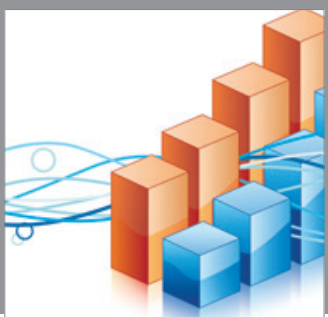

Advances in

Operations Research

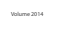

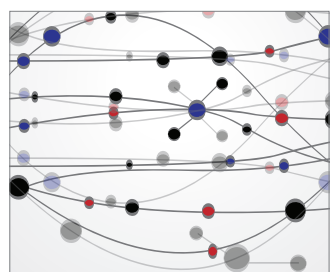

\section{The Scientific} World Journal
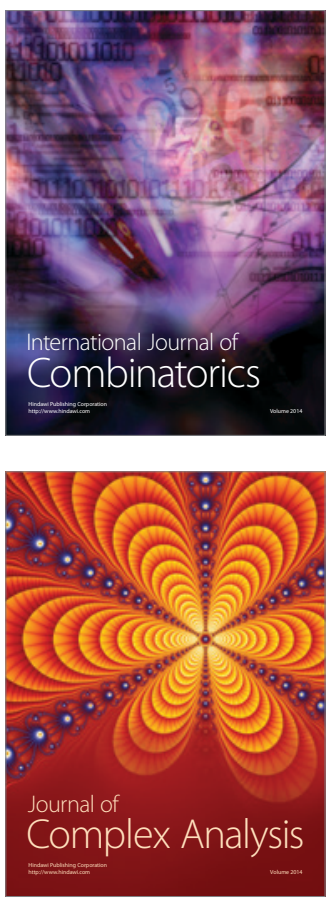

International Journal of

Mathematics and

Mathematical

Sciences
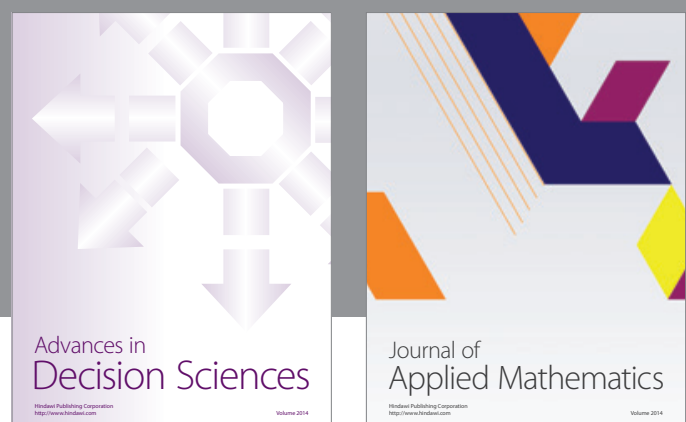

Journal of

Applied Mathematics
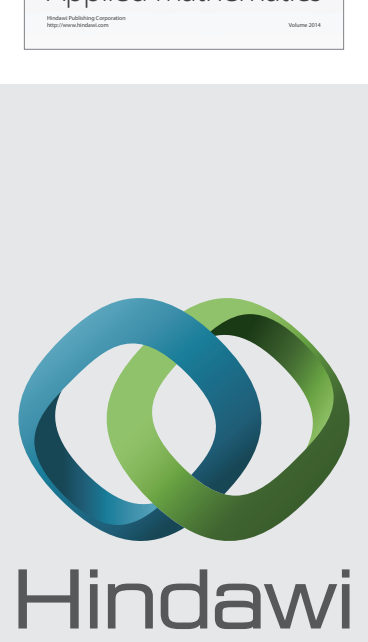

Submit your manuscripts at http://www.hindawi.com
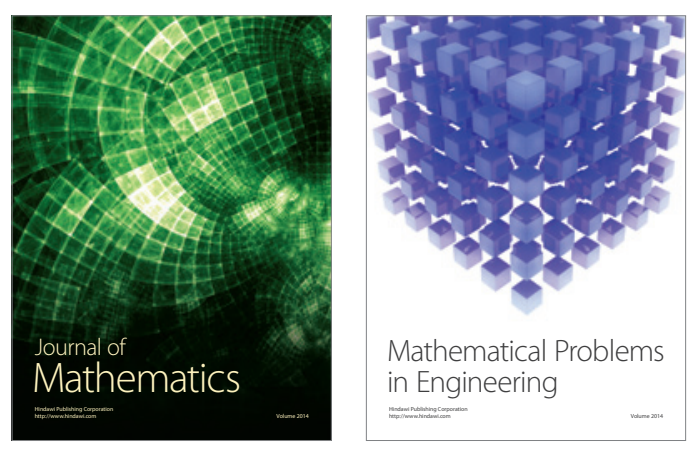

Mathematical Problems in Engineering
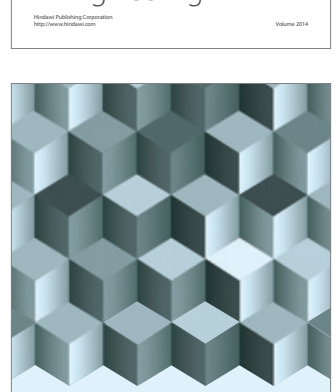

Journal of

Function Spaces
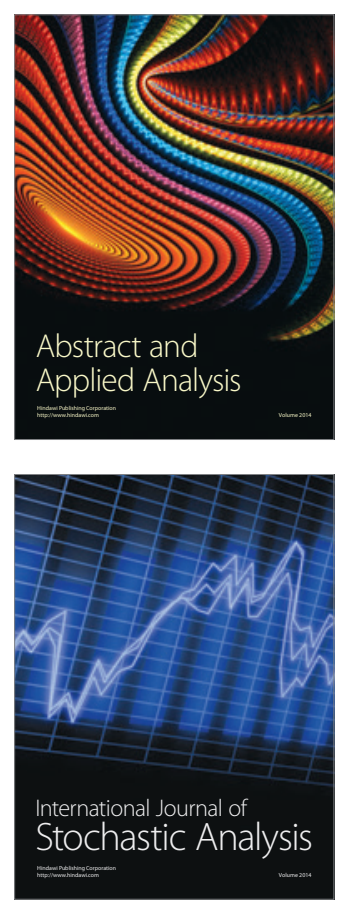

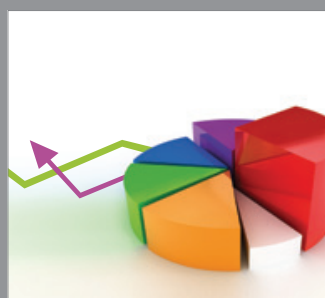

ournal of

Probability and Statistics

Promensencen
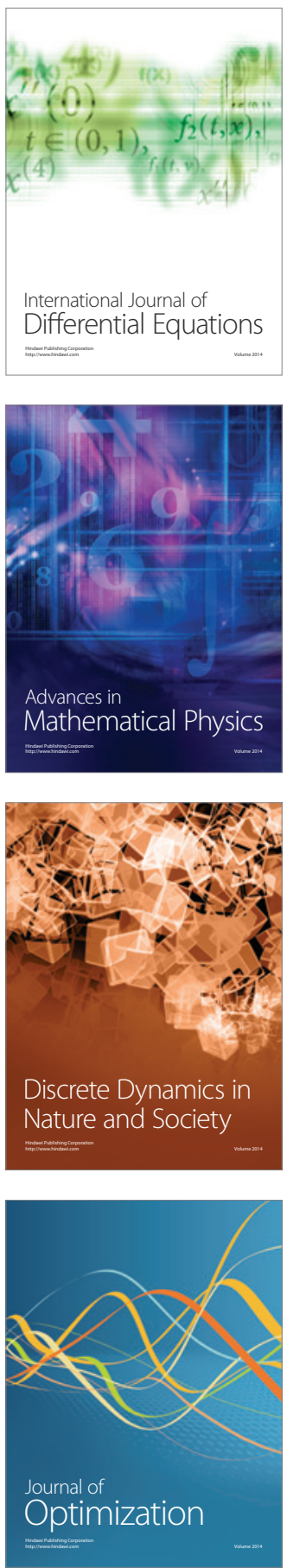